\title{
ON THE OBSERVATION OF PARITY ADMIXTURES WITH PERTURBED ANGULAR CORRELATIONS
}

\author{
R. R. LEWIS Jr. \\ Department of Physics, The University of Michigan, \\ Ann Arbor, Michigan ${ }^{\dagger}$
}

Received 29 February 1968

\begin{abstract}
A new parity test is proposed using combined electric and magnetic perturbation of the angular correlation of two gamma rays, one pure and one mixed. The experiment requires a measurement of the asymmetry of the coincidence rate in fixed counters for reversal of an applied magnetic field. An asymmetry of order $10^{-4}$ is predicted for ${ }^{181} \mathrm{Ta}$ in Hf. Either a single crystal or a powdered source may be used.
\end{abstract}

Considerable effort has gone into measurements of the circular polarization of nuclear gamma rays ${ }^{1}$ ) as one way of observing an admixture of multipole fields with opposite parity. The author has proposed an alternative experiment ${ }^{2}$ ) sensitive to the same admixture but using instead the perturbed angular correlation of two gamma rays, one pure and one mixed. Both experiments are designed to test the predictions of the current-current model of weak interactions ${ }^{3}$ ). The first experiment only requires measurement of the asymmetry in a single counter under reversal of a magnetic field, which can be performed to high precision. It has the disadvantage of a low efficiency of the polarimeter for detecting circular polarization. The second experiment involves measurement of a directional correlation and requires the precise reversal of either a counter or a crystalline axis. It is not clear whether this can be done to the desired accuracy.

In this work we shall suggest yet another experiment which appears to have all of the advantages of the first two experiments and fewer disadvantages. It requires the measurement of an angular correlation perturbed by both magnetic and electric fields. Under suitable conditions, the terms resulting from the interference of multipoles with like parity will be even in $\boldsymbol{H}$, whereas unlike parities lead to terms odd in $\boldsymbol{H}$. Therefore, the experiment requires measurement of an asymmetry of coincidence rates for fixed counters and source under reversal of $\boldsymbol{H}$

$$
\frac{W\left(\boldsymbol{k}_{1}, \boldsymbol{k}_{2}, \boldsymbol{H}\right)-W\left(\boldsymbol{k}_{1}, \boldsymbol{k}_{2},-\boldsymbol{H}\right)}{W\left(\boldsymbol{k}_{1}, \boldsymbol{k}_{2}, \boldsymbol{H}\right)+W\left(\boldsymbol{k}_{1}, \boldsymbol{k}_{2},-\boldsymbol{H}\right)} .
$$

These same terms are odd in $k_{2}$ (the direction of the mixed gamma ray), and thus the

$\uparrow$ Research sponsored by AFSOR under grant No. AF-AFOSR 1288-67. 
opposite asymmetry should be observed in a different pairs of counters

$$
\frac{W\left(\boldsymbol{k}_{1},-\boldsymbol{k}_{2}, \boldsymbol{H}\right)-W\left(\boldsymbol{k}_{1},-\boldsymbol{k}_{2},-\boldsymbol{H}\right)}{W\left(\boldsymbol{k}_{1},-\boldsymbol{k}_{2}, \boldsymbol{H}\right)+W\left(\boldsymbol{k}_{1},-\boldsymbol{k}_{2},-\boldsymbol{H}\right)}
$$

We conclude that perturbed correlations of gamma rays in single crystals (or in powdered sources) appear to offer a good alternative to polarization measurements of single gamma rays. The most interesting example is probably the $133 \mathrm{keV}-482 \mathrm{keV}$ cascade of ${ }^{181} \mathrm{Ta}$ in $\mathrm{Hf}$ metal ${ }^{4}$ ); weak interaction theory predicts an asymmetry of $\approx 1 \times 10^{-4}$ for a magnetic field of about $20 \mathrm{kG}$.

The experiment can be analysed with the standard formulation of perturbed angular correlations ${ }^{5}$ ). As a first example, consider a magnetic field parallel to an axially symmetric electric quadrupole field. The time-dependent correlation function is

$$
W\left(\boldsymbol{k}_{1}, \boldsymbol{k}_{2}, t\right)=\sum_{l_{1} l_{2}} \frac{4 \pi a_{l_{1}} b_{l_{2}}}{\sqrt{\left(2 l_{1}+1\right)\left(2 l_{2}+1\right)}} \sum_{q} Y_{l_{1}}^{q^{*}}\left(\boldsymbol{k}_{1}\right) G_{l_{1} l_{2}}^{q q}(t) Y_{l_{2}}^{q}\left(\boldsymbol{k}_{2}\right)
$$

The parameter $G$ is given by

$$
G_{l_{1} l_{2}}^{q q}(t)=\sqrt{\left(2 l_{1}+1\right)\left(2 l_{2}+1\right)} \sum_{m_{1} m_{2}}\left(\begin{array}{ccc}
I & I & l_{1} \\
m_{2} & -m_{1} & q
\end{array}\right)\left(\begin{array}{ccc}
I & I & l_{2} \\
m_{2}-m_{1} & q
\end{array}\right) \mathrm{e}^{-i\left(E_{m_{1}}-E_{m_{2}}\right) t}
$$

where the nuclear energy eigenvalues are

$$
E_{m}=m \omega_{\mathrm{H}}+\left[3 m^{2}-I(I+1)\right] \omega_{\mathrm{E}} \text {. }
$$

The notation is a standard one ${ }^{6}$ ) in which the Larmor frequency is $\omega_{\mathrm{H}}=g \mu_{0} H$, and the electric quadrupole frequency is $\omega_{\mathrm{E}}=e Q \Phi_{z z} / 4 I(2 I-1)$. Eq. (2) can be rewritten in the simpler form

$$
G_{l_{1} l_{2}}^{q q}(t)=\mathrm{e}^{-i q \omega_{\mathrm{H}} t} \sum_{n} S_{n q}^{l_{1} l_{2}}\left(\cos n \omega_{0} t,-i \sin n \omega_{0} t\right)
$$

for $l_{1}+l_{2}$ even and odd, respectively. The coefficients $S$ have been defined and tabulated by Harris ${ }^{7}$ ), and the frequency $\omega_{0}$ is $6 \omega_{\mathrm{E}}$ (or $3 \omega_{\mathrm{E}}$ ) for $I$ half-integer (or integer). Notice that $G$ has both diagonal $\left(l_{1}=l_{2}\right)$ and off-diagonal $\left(l_{1} \neq l_{2}\right)$ terms, which will be essential for the proposed experiment. It is the need for these off-diagonal terms which requires the quadrupole interaction; eq. (4) shows that $G$ has only diagonal terms in the limit $\omega_{0} \rightarrow 0$.

Now in order to devise a parity test calculate the real part of $G$

$$
\operatorname{Re} G_{l_{1} l_{2}}^{q q}=\left\{\begin{array}{lll}
\cos \left(q \omega_{\mathrm{H}} t\right) \sum_{n} S_{n q}^{l_{1} l_{2}} \cos \left(n \omega_{0} t\right) & \text { if } l_{1}+l_{2} \text { even } \\
-\sin \left(q \omega_{\mathrm{H}} t\right) \sum_{n} S_{n q}^{l_{1} l_{2}} \sin \left(n \omega_{0} t\right) & \text { if } l_{1}+l_{2} \text { odd }
\end{array}\right.
$$

which establishes our principal result

$$
\operatorname{Re} G_{l_{1} l_{2}}^{q q}(H)=(-)^{l_{1}+l_{2}} \operatorname{Re} G_{l_{1} l_{2}}^{q q}(-H)
$$


Combining the terms in the angular distribution coming from $q$ and $-q$ and using the hermiticity relation $G_{l_{1} l_{2}}^{q q^{*}}=G_{l_{1} l_{2}}^{-q-q}$, we obtain

$$
2 Y_{l_{1}}^{q}\left(\theta_{1} 0\right) Y_{l_{2}}^{q}\left(\theta_{2} 0\right)\left[\cos q\left(\phi_{1}-\phi_{2}\right) \operatorname{Re} G_{l_{1} l_{2}}^{q q}+\sin q\left(\phi_{1}-\phi_{2}\right) \operatorname{Im} G_{l_{1} l_{2}}^{q q}\right] \text {. }
$$

Hence if we choose $\boldsymbol{k}_{1}, \boldsymbol{k}_{2}$ and $\boldsymbol{H}$ coplanar $\left(\phi_{1}=\phi_{2}\right)$, the angular distribution becomes

$$
W\left(\boldsymbol{k}_{1}, \boldsymbol{k}_{2}, \boldsymbol{H}\right)=\sum_{l_{1} l_{2}} \frac{8 \pi a_{l_{1}} b_{l_{2}}}{\sqrt{\left(2 l_{1}+1\right)\left(2 l_{2}+1\right)}} \sum_{q \geqq 0} Y_{l_{1}}^{q}\left(\theta_{1} 0\right) Y_{l_{2}}^{q}\left(\theta_{2} 0\right) \operatorname{Re} G_{l_{1} l_{2}}^{q q},
$$

in which the terms even (odd) in $\boldsymbol{H}$ come from the terms of even (odd) parity. In particular, putting $\theta_{1}=\frac{1}{4} \pi, \theta_{2}=\frac{1}{2} \pi$ and dropping terms with $l \geqq 3$ for simplicity, we find

$$
W(\boldsymbol{H})=1+\sqrt{\frac{3}{4}} a_{2} b_{1} \operatorname{Re} G_{21}^{11}(\boldsymbol{H})+\frac{1}{8} a_{2} b_{2}\left[3 \operatorname{Re} G_{22}^{22}(\boldsymbol{H})-1\right]+\ldots
$$

The middle term is odd in $\boldsymbol{H}$ and proportional to $b_{1}$, which comes from the interference of multipole moments of opposite parity. Unlike the circular polarization, which contains only interference terms from multipoles of the same order, $b_{1}$ involves interference of all terms with multipole orders differing by 0 or 1 . This enhances $b_{1}$ by a factor $(\mathrm{E} 2 / \mathrm{M} 1) \approx 6 \mathrm{in}{ }^{181} \mathrm{Ta}$ relative to the circular polarization. The first and third terms in eq. (8) are even in $\boldsymbol{H}$ and give the ordinary perturbed anisotropy. Sample numerical results for the parameters $\operatorname{Re} G_{21}^{11}$ and $\operatorname{Re} G_{22}^{22}$ are shown in figs. 1 and 2 for $I=\frac{5}{2}$.

The derivation above confirms the general requirements of symmetry under inversions and rotations. The experiment proposed is in fact a direct test of symmetry under reflection in the plane of the counters. The directions $\boldsymbol{k}_{1}$ and $\boldsymbol{k}_{2}$ as well as the electric quadrupole field are all invariant under this reflection, but the magnetic field components in the plane are reversed. Since the quadrupole field is invariant under rotations through $\pi$ about the axis perpendicular to the plane, the consequences of this reflection symmetry are identical with the consequences of a spatial inversion. This explains why we have found relation (6) between terms odd in $\boldsymbol{H}$ and terms odd under inversions. Clearly the experiment must be designed to reduce any geometrical asymmetry which distinguishes "up" from "down" relative to this plane.

Next let us consider the consequences of relaxing some of the special assumptions made in the above derivation. First consider the effects of rhombic fields, which destroy the axial symmetry of the electric field gradients. The parameters $G$ are no longer diagonal in $q$, and eq. (1) becomes

$$
W\left(\boldsymbol{k}_{1}, \boldsymbol{k}_{2}, t\right)=\sum_{l_{1} l_{2}} \frac{4 \pi a_{l_{1}} b_{l_{2}}}{\sqrt{\left(2 l_{1}+1\right)\left(2 l_{2}+1\right)}} \sum_{q_{1} q_{2}} Y_{l_{1}}^{q_{1} *}\left(\boldsymbol{k}_{1}\right) G_{l_{1} l_{2}}^{q_{1} q_{2}}(t) Y_{l_{2}}^{q_{2}}\left(\boldsymbol{k}_{2}\right) .
$$

Although we can no longer write such explicit formulae for $G$ as eqs. (3)-(5), it can be shown that a symmetry like eq. (6) is still valid

$$
\operatorname{Re} G_{l_{1} l_{2}}^{q_{1} q_{2}}(H)=(-)^{l_{1}+l_{2}} \operatorname{Re} G_{l_{1} l_{2}}^{q_{1} q_{2}}(-H) .
$$


This follows from symmetry of the quadrupole field under two-fold rotations about the $x$-, $y$-and $z$-axes. However, we find that in order that $W$ contains only $\operatorname{Re} G$ we must now have $\boldsymbol{k}_{1}, \boldsymbol{k}_{2}$ and $\boldsymbol{H}$ coplanar, and in the $x$ - or $y$-plane of the rhombic fields.

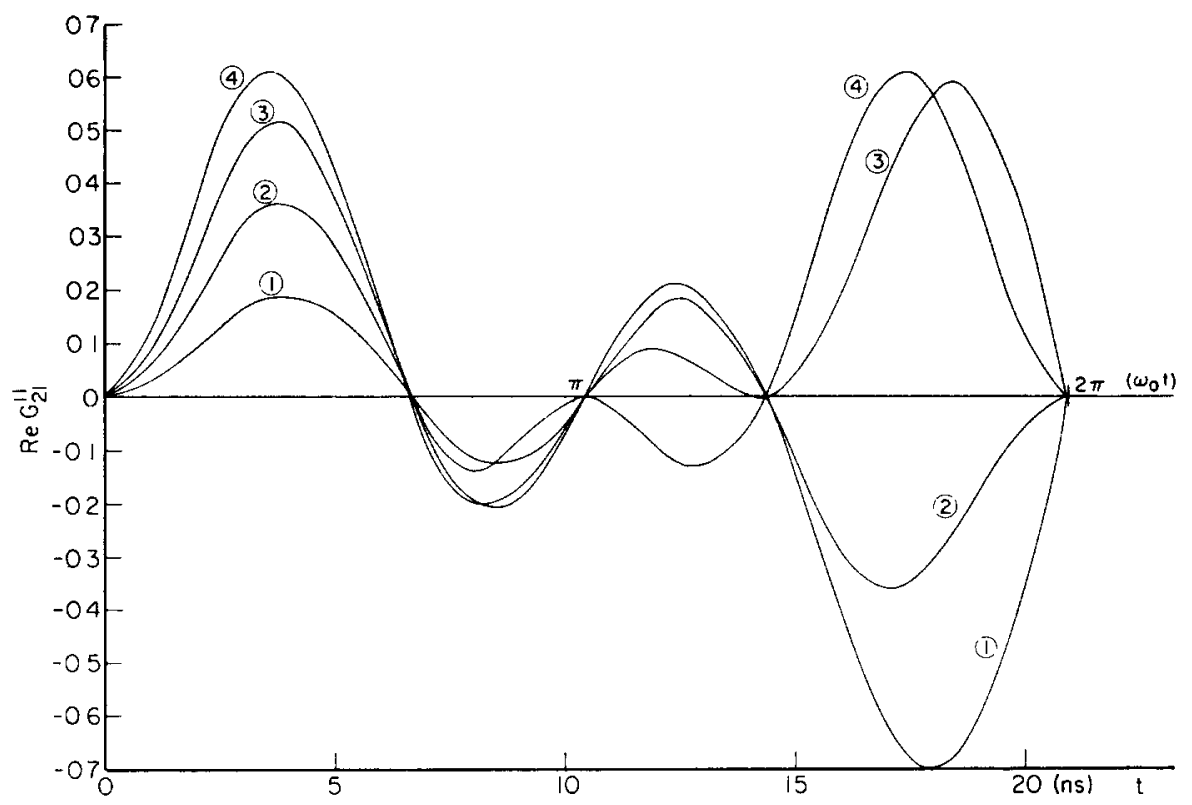

Fig. 1. The parameter $\operatorname{Re} G_{21}^{11}$ versus $\omega_{0} t$ for $I=\frac{5}{2}$ and for several different magnetic fields. The curves labelled 1, 2, 3, 4 correspond to $\left(\omega_{\mathrm{H}} / \omega_{0}\right)=\frac{1}{4}, \frac{1}{2}, \frac{3}{4}, 1$, respectively. This parameter is odd in both $\omega_{0}$ and $\omega_{\mathrm{H}} ; \omega_{0}$ was taken negative and $\omega_{\mathrm{H}}$ positive for this graph. For ${ }^{181} \mathrm{Ta}$ in $\mathrm{Hf}$, the magnitude of $\omega_{0}$ is known $\left.{ }^{4}\right)$ to be $\omega_{0}=(302 \pm 12) \times 10^{6} \mathrm{rad} / \mathrm{sec}$, but the sign is not known; $\omega_{\mathbf{H}}=\omega_{0}$ corresponds to a magnetic field of $46 \mathrm{kG}$ and $\omega_{0} t=2 \pi$ to $21 \mathrm{~ns}$.

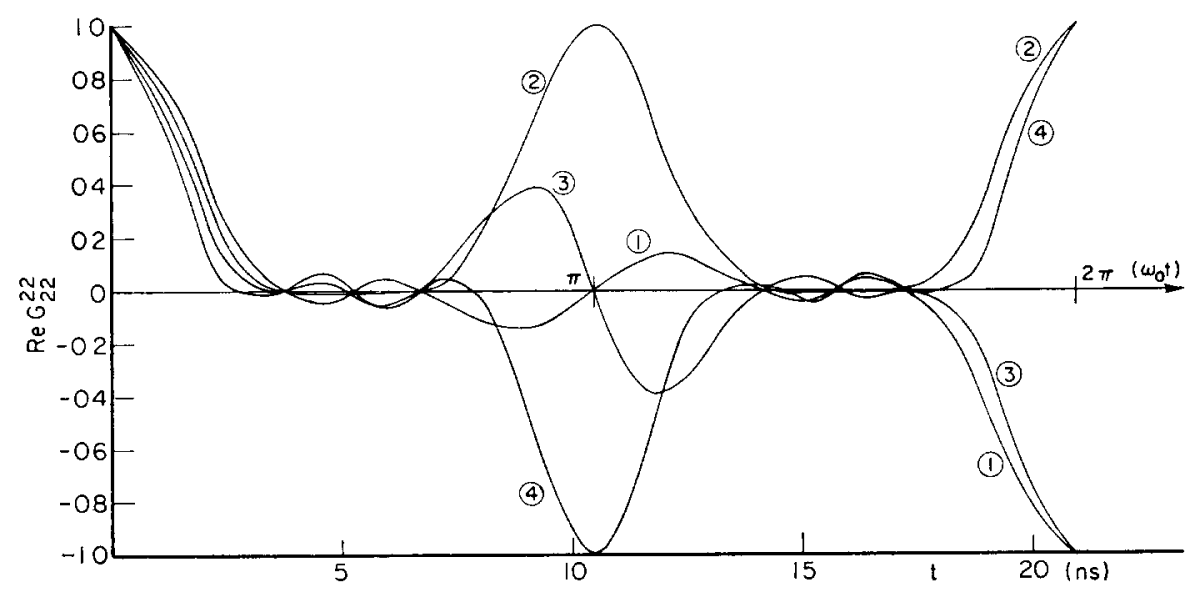

F1g. 2. The parameter $\operatorname{Re} G_{22}^{22}$ versus $\omega_{0} t$ for $I=\frac{5}{2}$. The notation is the same as for fig. 1. This parameter is independent of the sign of $\omega_{0}$ and $\omega_{\mathbf{H}}$. 
There are no calculations of $G$ available for this configuration. We conclude that rhombic fields would not in principle invalidate the parity test but would complicate the situation by demanding precise knowledge of the azimuthal orientation. Since, in any event, the experiment would require a single crystal to be of high perfection, it would be best to choose one with sufficient crystalline symmetry to avoid rhombic fields.

Another way of avoiding the effects of rhombic fields (or misalignment of the crystal) is to use a powdered crystalline source instead of a single crystal. It is easily shown that averaging over all azimuthal orientations of the microcrystalline axes leads to the same eq. (6); in the notation of Alder et al. ${ }^{5}$ ), we find $\left\langle G_{l_{1} l_{2}}^{q_{1} q_{2}}\right\rangle_{\gamma}$ had only diagonal elements in $q$, satisfying

$$
\operatorname{Re}\left\langle G_{l_{1} l_{2}}^{q q}(H)\right\rangle_{\gamma}=(-)^{l_{1}+l_{2}} \operatorname{Re}\left\langle G_{l_{1} l_{2}}^{q q}(-H)\right\rangle_{\gamma} .
$$

Since there is one less direction to determine, powdered sources are probably better than single crystals; the only disadvantage would be a possible decrease in the magnitude of $\operatorname{Re}\left\langle G_{12}^{11}\right\rangle_{\gamma}$ due to the averaging. No calculations are available for $l_{1} \neq l_{2}$, but the results for $l_{1}=l_{2}$ indicate $^{5}$ ) that the averaging reduces $\operatorname{Re}\left\langle G_{22}\right\rangle$ by about one half.

Next consider the effects of misalignment of the various directions. As eq. (7) shows, if either counter is out of the plane $\phi=0$, then there will be terms odd in $\boldsymbol{H}$ but even in parity. For example, there will be an even-parity contribution

$$
a_{2} b_{2} Y_{2}^{2}\left(\theta_{1} 0\right) Y_{2}^{2}\left(\theta_{2} 0\right) \sin 2\left(\phi_{1}-\phi_{2}\right) \operatorname{Im} G_{22}^{22}(H),
$$

which is odd in $\boldsymbol{H}$. It can be distinguished from the odd-parity terms like

$$
a_{2} b_{1} Y_{2}^{1}\left(\theta_{1} 0\right) Y_{1}^{1}\left(\theta_{2} 0\right) \cos \left(\phi_{1}-\phi_{2}\right) \operatorname{Re} G_{21}^{11}(H),
$$

by reversing $\boldsymbol{k}_{2}$ or by varying $\left(\phi_{1}-\phi_{2}\right)$. A similar analysis can be given for the effect of a small component of $\boldsymbol{H}$ out of the plane; misalignment of $\boldsymbol{H}$ in the plane does not influence the parity test.

We have based the discussion on the time-resolved coincidence rate. Time-averaged results will show the same effect, but the magnitude of $G$ is reduced by the timeaveraging. It would probably be best to choose a region of time delays where the signal is large; this can be done if $\omega_{0}$ and $\omega_{\mathrm{H}}$ are known. There is also the possibility of enhancing the signal by using its characteristic time dependence given by eq. (5). For $I=\frac{5}{2}, G_{12}^{11}$ has Fourier components with $n=1,2$, whereas $G_{22}^{22}$ has $n=1,3$. It is also amusing to note the possibility of a trick similar to that of Lobashov et al. ${ }^{1}$ ). If the coincidence rate is sufficiently high one can modulate the applied magnetic field at some low frequency, $\mathrm{H}=\mathrm{H}_{0} \cos \Omega t$. Then the coincidence rate (at some fixed angle and delay time) is modulated by

$$
\begin{array}{lll}
\operatorname{Re} G_{l_{1} l_{2}}^{q q} \sim \sin (q \Theta \cos \Omega t) & \text { if } & l_{1}+l_{2} \text { odd, } \\
\operatorname{Re} G_{l_{1} l_{2}}^{q q} \sim \cos (q \Theta \cos \Omega t) & \text { if } & l_{1}+l_{2} \text { even, }
\end{array}
$$


which shows that the odd-parity terms are modulated at odd harmonics of $\Omega$, whereas the even-parity terms are modulated at even harmonics.

The author gratefully acknowledges stimulating conversations on this subject with Professor W. Williams, Dr. J. C. Van der Leeden and with Dr. E. Kauffman.

\section{References}

1) F. Boehm and E. Kankeleit, Phys. Rev. Lett. 14 (1965) 312;

P. Bock and H. Schopper, Phys. Lett. 16 (1965) 284;

V. M. Lobashov et al., JETP Lett. 3 (1966) 47, 173, 5 (1967) 59

2) R. R. Lewis, Jr., Phys. Rev. Lett. 17 (1966) 593;

Phys. Rev. 163 (1967) 935

3) R. J. Blin-Stoyle, Phys. Rev. 118 (1960) 1605, 120 (1960) 181;

F. C. Michel, Phys. Rev. 133 (1964) B329

4) M. Salomon et al., Ark. Fys. 27 (1964) 97

5) R. M. Steffen and H. Frauenfelder, in Perturbed angular correlations, ed. by E. Karlsson, E. Matthias and K. Siegbahn (North-Holland Publ. Co., Amsterdam, 1964)

6) K. Alder, E. Matthias, W. Schneider and R. Steffen, Phys. Rev. 129 (1963) 1199

7) S. H. Harris, Nucl. Phys. 11 (1959) 387 